\title{
Médiévales
}

Langues, Textes, Histoire

\section{À qui profitent les guerres en Orient ? Quelques observations à propos des conflits entre Slaves et Francs au IX ${ }^{\mathrm{e}}$ siècle}

Who benefits from the Wars in the East? Some Observations about the Conflits between Slavs and Franks during the 9th Century

\section{Thomas Lienhard}

\section{(2) OpenEdition}

Journals

Édition électronique

URL : https://journals.openedition.org/medievales/1527

DOI : 10.4000/medievales. 1527

ISSN : 1777-5892

Éditeur

Presses universitaires de Vincennes

Édition imprimée

Date de publication : 1 décembre 2006

Pagination : 9-84

ISBN : 978-2-84292-193-4

ISSN : 0751-2708

\section{Référence électronique}

Thomas Lienhard, «À qui profitent les querres en Orient? Quelques observations à propos des conflits entre Slaves et Francs au ix ${ }^{\mathrm{e}}$ siècle », Médiévales [En ligne], 51 | automne 2006, mis en ligne le 02 novembre 2010, consulté le 23 avril 2022. URL : http://journals.openedition.org/medievales/1527 ; DOI : https://doi.org/10.4000/medievales.1527

Ce document a été généré automatiquement le 23 avril 2022

Tous droits réservés 


\title{
À qui profitent les guerres en Orient? Quelques observations à propos des conflits entre Slaves et Francs au IX ${ }^{\mathrm{e}}$ siècle
}

\author{
Who benefits from the Wars in the East? Some Observations about the Conflits \\ between Slavs and Franks during the 9th Century
}

Thomas Lienhard

Dans le dernier tiers du viII ${ }^{\mathrm{e}}$ siècle, les Carolingiens imposèrent leur domination à la fois en Saxe, en Bavière et dans le nord de la péninsule italienne. Ce déplacement des frontières inversa la situation des Francs au sein du monde occidental : naguère objets d'intégration au sein du monde romain, ils se voyaient désormais tenus d'administrer à leur tour des régions récemment conquises, et de contrôler ou de soumettre des nouveaux voisins en Europe centrale. Pourtant, Aix n'était pas Rome : là où l'empire antique avait constitué un pôle d'attraction et d'insertion à tel point qu'aucun peuple durable ne s'était développé en-dehors du limes, l'ère franque vit au contraire se développer les premiers peuples slaves en dehors du système carolingien, et même en opposition à ce dernier. Faut-il en conclure que ces populations slaves disposaient déjà, à l'aube $d u{ } x^{e}$ siècle, d'un potentiel identitaire plus fort que celui des barbares antiques, qui les aurait détournées du monde franc? Pour apprécier et pour expliquer l'attitude des Carolingiens sur leurs frontières orientales, il est indispensable d'étudier à la fois les modalités franques d'administration et le degré d'autonomie et d'identité ethnique des populations concernées.

2 Or qui veut décrire l'histoire de l'identité slave n'est pas seul. On pourrait même dire que ce thème, fort débattu par les médiévistes depuis deux siècles, en est actuellement à son troisième âge dans l'historiographie; dans la mesure où il s'agit là d'un facteur d'explication déterminant pour la politique orientale des Francs, il ne sera peut-être pas inutile de rappeler brièvement ici l'histoire de ce concept. Une première école, née 
au XIX ${ }^{e}$ siècle et dominante jusqu'à la Seconde guerre mondiale, prêtait aux peuples slaves une stabilité et une ancienneté extrêmement marquées : dès leur installation en Europe, que cette historiographie datait $\mathrm{du} \mathrm{I}^{\mathrm{er}}$ siècle de notre ère, ces populations auraient présenté des spécificités ethniques suffisantes (Ernst Dümmler parlait d'« esprit ») pour déclencher tout naturellement des conflits d'intérêt avec les peuples occidentaux, et notamment avec les Francs lorsque ceux-ci se tournèrent vers l'est ${ }^{1}$. Cette présentation des événements fut ébranlée à partir des années 1960 par une deuxième école historiographique : celle-ci dut son essor à Reinhard Wenskus, qui proposait une autre explication pour la naissance des peuples médiévaux, et fut appliquée au domaine slave par Herwig Wolfram ${ }^{2}$. Elle soulignait désormais les aspects exclusivement sociaux, c'est-à-dire fluctuants et négociables, de l'identité ethnique : ce fut progressivement, en accumulant des facteurs tels qu'un ethnonyme stable, une vie religieuse commune ou un pouvoir politique fédérateur, que les Slaves en vinrent à constituer des peuples. Cet acquis conceptuel, s'il ne fut jamais remis en cause dans ses grandes lignes, a pourtant appelé à son tour quelques compléments qui ont permis le rassemblement d'une troisième école d'historiens ${ }^{3}$; en particulier, on chercha désormais à préciser la causalité de ce processus. Pour Wenskus et Wolfram, l'accumulation de facteurs ethniques qui avait engendré les peuples médiévaux résultait du seul hasard historique, par une génération lente et spontanée ; à l'opposé, on fit valoir plus récemment que diverses autorités médiévales avaient pu déclencher ou accélérer ce phénomène. En particulier, les Francs, qui furent les principaux témoins de ce processus social chez les Slaves, n'ont-ils pas canalisé cette évolution en fonction de leur propre passé et dans le sens de leurs propres intérêts? Dans la naissance dont Wenskus a montré les étapes, il est loisible de chercher l'accoucheur.

Telle est la problématique que l'on souhaiterait appliquer ici à deux épisodes exemplaires pour les origines des peuples slaves. Il s'agit, dans un cas, des conflits répétés qui, entre 815 et 823 , mirent en présence les armées de Louis le Pieux et les Slaves, à la fois sur l'Elbe et en Pannonie; le second exemple vit s'opposer, durant quelque quarante ans, les premiers princes de Moravie et les forces de Francie orientale dans la seconde moitié du IX ${ }^{e}$ siècle. Le dossier documentaire qui permet d'analyser ces événements est relativement simple: on ne fera référence qu'aux textes annalistiques $\mathrm{du} \mathrm{IX}^{\mathrm{e}}$ siècle et à une lettre rédigée par un comte franc, Arbo, qui avait été dépêché auprès des Moraves vers 890 . Bien connus par les historiens, ces trois textes présentent en outre l'avantage d'une excellente tradition manuscrite, puisque la lettre d'Arbo est conservée sous sa forme originale et que les annales sont transmises par des manuscrits qui datent, au pire, de cinquante ans après la conception originale du texte : le risque d'une interpolation se voit ainsi réduit, étant entendu que cela ne préjuge en rien du crédit que l'on peut accorder à l'auteur. 


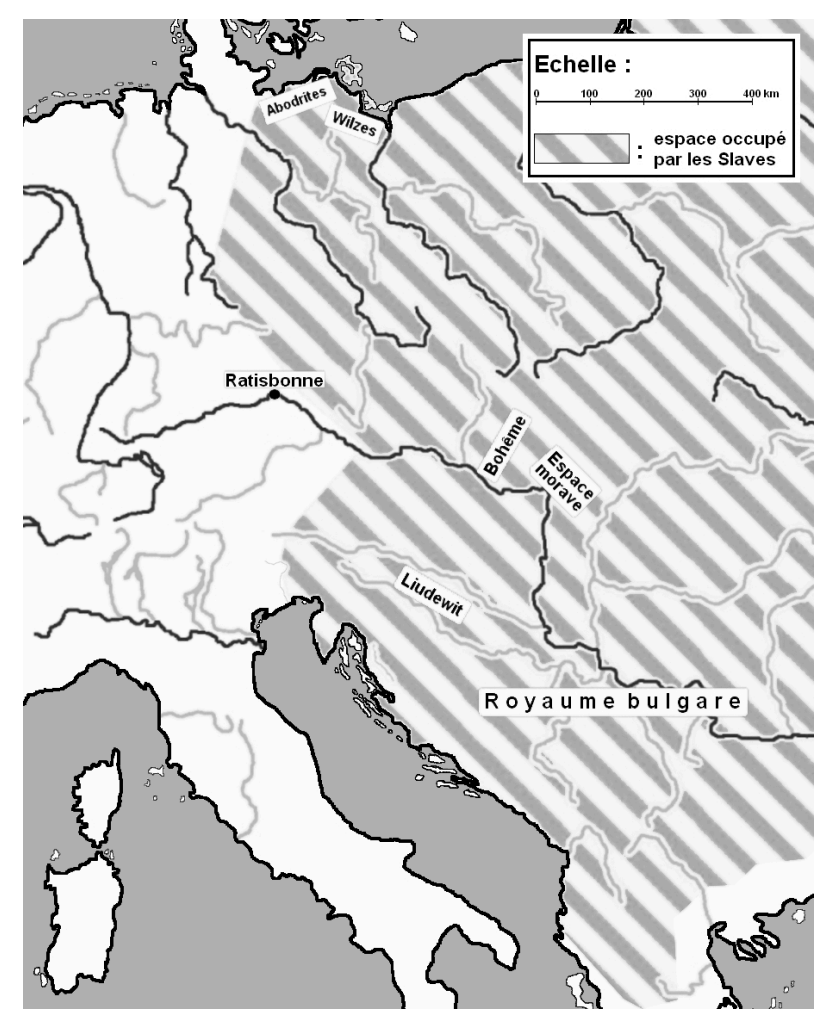

4 Le dossier est vaste, tant par la longue période qu'il envisage que par le poids historiographique qu'il véhicule. C'est pourquoi on se contentera ici, au risque de paraître allusif, d'une seule question : lors de ces tensions, les Francs ont-ils subi, ou au contraire suscité le développement des mouvements identitaires slaves? L'argumentation procédera en trois temps: on s'efforcera d'abord de montrer qu'au début de chaque conflit, les facteurs de cohésion ethnique étaient encore fort maigres chez les populations concernées: il n'y avait pas encore d'ensemble politique nettement constitué chez les Slaves du nord-ouest, ni en Pannonie, au début du $\mathrm{IX}^{\mathrm{e}}$ siècle, et l'historien ne distingue pas davantage les bases d'une autonomie morave avant les années 850. Dans ces conditions, comment interpréter la soudaine stabilité qui, à partir de ces périodes-charnières, fut attribuée à ces populations par les sources latines? On entrera alors davantage dans le détail de ces deux conflits, pour mettre en lumière l'attitude et les intérêts de ceux qui, du côté franc, furent à la fois les principaux acteurs vis-à-vis des Slaves et les informateurs prioritaires pour les auteurs de nos sources; le dossier des Slaves de Pannonie, étudié dans ce deuxième temps, permettra de proposer une hypothèse qui pourrait paraître fragile étant donné le laconisme des annales royales. C'est pourquoi on terminera la présente étude en exploitant le dossier morave, mieux documenté, dans l'espoir de dégager une causalité commune dans ces deux cas de naissance ethnique.

\section{Des peuples slaves spécifiques avant 815 ? Les historiens en quête de critères}

Pourquoi remettre en question l'existence de peuples slaves à l'aube du $\mathrm{IX}^{\mathrm{e}}$ siècle ? Les sources semblaient pourtant catégoriques, qui, d'année en année, employaient des ethnonymes constants pour caractériser les événements en Europe centrale : ainsi les 
« Abodrites », nommés pour la première fois en $789^{4}$, apparurent avec régularité dans les textes durant les siècles suivants. Pour le lecteur médiéval comme pour l'historien moderne, cette répétition pouvait produire une impression rassurante: s'il y avait ethnonyme, c'était qu'il y avait peuple. Il semblait donc possible d'élaborer une géographie ethnique de l'Europe centrale, et par là d'appréhender cet espace mal connu.

6 Encore faut-il rappeler à ce propos un trait caractéristique des auteurs du haut Moyen Âge $^{5}$ : à la différence des modernes, les ethnographes carolingiens concevaient difficilement un processus de mutation interne au sein d'un ensemble ethnique. Dans cette optique, un peuple naissait brutalement, par fragmentation à partir d'un des peuples originels ; il pouvait éventuellement connaître une migration, ou être anéanti par les armes; en revanche, on envisageait rarement d'autres facteurs de variation ethnique, tels que la fusion au sein d'un nouvel ensemble ou l'absorption, au contraire, de nouvelles populations par la construction d'une culture commune. Par conséquent, la régularité des ethnonymes appliqués aux Slaves par les Carolingiens peut refléter les conceptions immobilistes d'un auteur, autant qu'une quelconque réalité sociale : si un personnage venu d'Europe centrale s'était attribué (ou : vu attribuer, même par erreur) un ethnonyme déterminé, cela semblait suffisant pour faire admettre l'existence, dans ces régions mal identifiées, d'un peuple durable dont les auteurs latins allaient ensuite employer le nom durant des décennies, même si aucun groupe stable ne s'y était développé. De ce fait, pour démontrer la cohérence d'ethnies slaves vers 800 , l'historien devait rechercher non seulement des noms, mais également des pratiques sociales stables et spécifiques, qui auraient distingué un groupe d'un autre. Trois voies furent explorées dans cet objectif: l'archéologie, la géographie politique et les pratiques religieuses.

7 Le recours aux données archéologiques fut d'autant plus important que, dans les secteurs considérés, le patrimoine était considérable et fut patiemment mis en lumière par les archéologues de la RDA puis par leurs successeurs, ainsi que par les chercheurs hongrois, slovènes ou croates ${ }^{6}$. Or précisément, parmi les données découvertes, des variantes régionales semblaient renforcer l'hypothèse d'ensembles culturels distincts, dont l'archéologie aurait pu éclairer l'histoire et même la préhistoire : ces chercheurs évoquaient ainsi la structure et la vie politique des groupes sorabes, abodrites ou croates avant même la mention de ces derniers dans les sources écrites vers $800^{7}$. Depuis quelque deux décennies toutefois, les critiques se sont multipliées envers ce premier argument. D'une part, en effet, celui-ci nécessitait d'admettre l'existence d'une relation, toujours délicate à établir, entre culture matérielle et structure politique. D'autre part, les historiens qui défendaient cette classification durent postuler la rareté des échanges entre les groupes concernés: dans cette conception, les données archéologiques découvertes auraient été le fruit d'un développement autonome au sein $\mathrm{du}$ groupe slave en question, et non pas le simple résultat d'échanges permanents le long de la mer Baltique ou de la côte dalmate. Or un tel point de vue fut largement remis en cause par des études plus récentes dont les auteurs constataient, au contraire, une abondante circulation de biens matériels entre les groupes sociaux de ce vaste espace ${ }^{8}$. Dans le cas des Slaves à l'époque carolingienne, force fut donc de constater que les aires archéologiques avaient des frontières fort perméables, et de renoncer à cet argument pour étayer l'existence de structures politiques distinctes. 
Une deuxième hypothèse consista à admettre que les groupes slaves se seraient développés de façon distincte en fonction de leur localisation par rapport au monde franc: si les Carolingiens entretenaient des relations pacifiques avec certains groupes slaves, tels que les Abodrites, mais multipliaient les conflits avec d'autres, tels que les Wilzes, n'était-ce pas parce que les seconds étaient plus éloignés, et par là même moins imprégnés par la culture franque que les premiers? Séduisante par sa simplicité, cette hypothèse doit pourtant être écartée pour plusieurs raisons. Avant tout, les textes du $\mathrm{IX}^{\mathrm{e}}$ siècle ne nous renseignent guère à propos de la localisation des Wilzes : c'est donc sur des mentions plus tardives, généralement postérieures au $\mathrm{x}^{\mathrm{e}}$ siècle, que s'appuie cette suggestion. De plus, quel que fût l'espace occupé par les Wilzes, il était moins éloigné des Francs, en tout état de cause, que d'autres aires avec lesquelles les Carolingiens étaient pourtant en mesure d'échanger régulièrement en ce début du $\mathrm{IX}^{\mathrm{e}}$ siècle ${ }^{9}$. Les Wilzes eux-mêmes, d'ailleurs, entretenaient avec les Francs des relations certes belliqueuses, mais tout aussi fréquentes que leurs voisins abodrites ${ }^{10}:$ pas plus que la culture matérielle, la localisation géographique ne semble donc avoir constitué un facteur décisif pour la spécificité d'un peuple slave.

Sans doute est-ce donc le critère religieux qui conserve le plus de pertinence. En effet, la présence de personnages non baptisés, tels que le chef abodrite Sclaomir nommé par les Annales du royaume des Francs ${ }^{11}$, est encore attestée parmi les Slaves de l'ouest au début du IX $\mathrm{X}^{\mathrm{e}}$ siècle. Or étant donnée l'importance du christianisme dans la vie publique carolingienne, il semble évident que cet écart religieux n'était pas de nature à faciliter les échanges entre les Francs et les païens en question, contribuant ainsi à isoler au moins une partie des Slaves sur le plan diplomatique. Toutefois, même ce dernier argument exige deux rectificatifs. D'une part en effet, l'élément religieux, s'il était assurément crucial dans la société médiévale, ne fut pourtant jamais invoqué par l'auteur des annales pour justifier les offensives franques contre un groupe slave: le texte se contentait tout au plus de dénoncer, en termes très généraux, la "rébellion » d'un dirigeant local. D'autre part, les premiers empereurs carolingiens ne répugnèrent pas à traiter avec des dirigeants païens en cas de besoin : le chef païen Sclaomir, évoqué à l'instant, ne fut-il pas placé à la tête des Abodrites par les Francs eux-mêmes, gouvernant durant cinq ans avec la bénédiction de ces derniers sans jamais se voir imposer un baptême? Ainsi, et quelque décisive que fût l'appartenance religieuse par ailleurs, celle-ci ne suffisait manifestement pas pour précipiter la cristallisation d'un peuple slave en l'acculant au conflit contre les Francs ou contre d'autres voisins.

Plus généralement, aucun des critères examinés jusqu'ici ne plaide pour une opposition de type identitaire entre deux cultures différentes. Il importe donc, en deuxième partie de cette étude, d'aborder autrement la relation de causalité entre les origines des peuples slaves (car ceux-ci, dans le courant du IX ${ }^{e}$ siècle, s'affirment incontestablement) et les tensions récurrentes dans ces espaces.

\section{Les Slaves de Pannonie : ignorance et dépendance des Carolingiens face à leurs relais locaux}

Pour comprendre le déclenchement des conflits à l'est de l'espace franc, il n'est sans doute pas inutile de présenter la diversité des acteurs au sein du monde carolingien. Il serait erroné, en effet, de considérer que celui-ci se comporta face aux Slaves comme 
un ensemble uniforme ; bien au contraire, la multiplicité des autorités occidentales, et la compétition qui régnait parfois entre elles, semble avoir constitué un aiguillon d'importance pour les évolutions sociales qui, au IX siècle, affectèrent les frontières orientales du monde franc.

Si la politique carolingienne ne fut pas homogène en ce domaine, ce fut notamment parce que le pouvoir central faisait preuve d'une méconnaissance étonnante à propos du monde slave; les exemples de cette ignorance ne manquent pas. Ainsi, en 822, l'auteur des Annales du royaume des Francs présentait en une même phrase les Abodrites et les Praedenecenti, qui apparaissaient alors pour la première fois dans nos sources et qu'il situait entre les Moraves et les Avars ${ }^{12}$. À cette localisation exacte, il en substituait une autre deux ans plus tard: il précisait en effet que les Praedenecenti se trouvaient " en Dacie, près du Danube, aux côtés des Bulgares ", et considérait également que ce groupe et celui des Abodrites ne constituaient qu'un seul et même peuple ${ }^{13}$. Or cette dernière affirmation semble totalement invraisemblable, puisqu'elle obligerait à déplacer la localisation des Abodrites de quelque deux mille kilomètres par rapport à toutes les autres indications fournies par nos sources: manifestement, l'auteur s'était quelque peu embrouillé, peut-être parce que pour l'année 822, les Annales avaient présenté ces deux peuples presque immédiatement à la suite l'un de l'autre.

Un autre exemple d'une incertitude similaire se déroula en 839 : cette année-là, selon les Annales de Saint-Bertin, "L'empereur Théophile envoya également quelques hommes qui se nommaient, ou qui nommaient le peuple auquel ils appartenaient, par le terme de "Rhos", et qui affirmaient que leur roi, appelé Khagan, les avait envoyés vers lui [Théophile] pour des raisons d'amitié ; il demandait que, par la bienveillance de l'empereur [Louis le Pieux], on leur accordât l'autorisation et l'assistance nécessaires pour rentrer chez eux à travers son empire [...]. L'empereur [Louis] fit faire une enquête plus précise au sujet de la cause de leur venue, et apprit qu'ils appartenaient au peuple des Suédois; considérant qu'ils étaient plutôt venus comme éclaireurs dans notre royaume que pour rechercher la paix, il ordonna de les retenir auprès de lui jusqu'à ce qu'on eût déterminé s'ils étaient venus en toute bonne foi ou non ${ }^{14}$ ».

Quelle que fût l'origine réelle de ces voyageurs, un point fondamental réside dans l'incertitude manifestée par le pouvoir carolingien. Certes, l'annaliste semblait suggérer la présence d'une équipe d'experts efficaces auprès de l'empereur, puisqu'il évoquait une enquête qui aurait produit un résultat ferme; toutefois, l'hésitation ultérieure de Louis semblait contredire cette présentation, et donnait à comprendre qu'à la cour franque, dans la première moitié du IX siècle, on manquait cruellement d'informations pour mener une politique slave.

Dans ces conditions, le monopole du renseignement revenait aux représentants locaux du pouvoir carolingien. Ainsi, quand il fallut déterminer la politique à adopter face aux Bulgares en 826, l'empereur fit envoyer à plusieurs reprises des émissaires auprès de Balderich, préfet du Frioul, qui était manifestement le seul à pouvoir l'éclairer ${ }^{15}$. De même, lorsque les Francs obtenaient, dans ces espaces-frontières, l'alliance militaire d'un chef slave, celui-ci devenait leur unique informateur à propos des campagnes qu'il menait: «le déroulement de ces opérations, il le fit raconter à l'empereur par l'intermédiaire de ses envoyés ", écrivait l'annaliste à propos de l'un d'entre eux, comme pour décliner toute responsabilité à propos de la vérité du récit ${ }^{16}$. On aura garde de ne pas oublier ce point: pour composer son texte, l'auteur n'avait d'autre choix que de retranscrire fidèlement les éléments que lui transmettaient ces 
informateurs locaux, qui s'efforçaient pour leur part de promouvoir leurs intérêts particuliers. Il y a là un prisme de lecture dont il faut tenir compte avant d'interpréter les relations sociales dans les périphéries carolingiennes.

C'est cette précaution qui amène à reconsidérer le conflit majeur du début du règne de Louis le Pieux, celui qui opposa les armées franques et le chef slave Liudewit, qualifié de dux de Pannonie inférieure. D'année en année, entre 819 et 822, celui-ci fut l'objet des assauts francs, suscitant des inquiétudes suffisantes pour que fussent dirigés contre lui les trois corps d'armée mobilisés pour les opérations principales. Pour expliquer la guerre, l'annaliste se montra fort évasif, n'évoquant que la trahison (defectio) du Pannonien ${ }^{17}$. L'historiographie a choisi d'interpréter ces événements comme un conflit entre deux groupes culturels : en ce IX ${ }^{e}$ siècle qui, de fait, vit naître les premiers peuples durables parmi les Slaves du sud ${ }^{18}$, Liudewit aurait représenté le refus, par un de ces peuples naissants, d'accepter l'hégémonie franque ${ }^{19}$. Toutefois, les modalités précises du conflit confirment-elles cette perspective? Certes, dès son apparition dans les sources, le chef slave se plaignit des abus de pouvoir pratiqués, selon lui, par les représentants francs dans le sud-est ${ }^{20}$. Il est toutefois frappant de constater que pour cela, loin de rejeter l'influence carolingienne dans son ensemble, il exprima au contraire sa plainte devant le plaid impérial, et n'eut de cesse, dans les années suivantes, de joindre l'empereur directement, en essayant d'éviter le truchement des chefs francs locaux ${ }^{21}$; il ne prit d'ailleurs aucune initiative militaire contre les Francs, se contentant de reculer progressivement au rythme de la progression de ses agresseurs. Compte tenu de ces étrangetés dans le comportement de Liudewit, une autre hypothèse semble possible pour expliquer ce conflit: le chef pannonien ne cherchait nullement à préserver un quelconque peuple slave face au monde carolingien, mais à limiter le pouvoir personnel des Francs locaux. Pour leur part, ces derniers défendirent leur position en présentant, grâce au monopole d'information dont ils disposaient, Liudewit comme un rebelle dont l'activité menaçait tout le monde franc. Si l'on accepte ce schéma, ce n'est donc pas tant un mouvement identitaire slave qui explique la guerre, mais la contestation du pouvoir comtal, en Pannonie comme ailleurs dans le monde franc; dans ce secteur périphérique toutefois, les tensions prirent une ampleur extrême parce que l'arbitraire du comte, en raison de l'éloignement géographique, était plus difficile à contrôler.

\section{La Moravie : rébellion des Slaves, ou des Francs installés sur les frontières?}

17 L'interprétation proposée à l'instant pourrait paraître hasardeuse si l'on ne disposait que de ce premier dossier : en effet, les Annales du royaume des Francs, si elles sont plus détaillées à propos de Liudewit que pour d'autres conflits, restent pourtant avares de précisions, et bien des points de ce conflit demeurent dans l'ombre. En revanche, un même schéma triangulaire, mettant en présence à la fois un pouvoir central carolingien, des relais locaux et des groupes slaves, se retrouve également dans le cas de l'ensemble morave, dont les premiers pas sont mieux documentés. En l'occurrence, on assiste incontestablement à l'apparition d'un peuple dont la présence et l'unité n'allaient plus se démentir jusqu'à l'époque moderne. Celui-ci constitua d'ailleurs pour les Francs un adversaire redoutable, puisque les conflits entre ces deux ensembles s'étendirent sur toute la seconde moitié $\mathrm{du} \mathrm{IX}^{\mathrm{e}}$ siècle, avec un rythme proche d'une 
campagne par an ${ }^{22}$. Faut-il en conclure que la guerre fut le prix de cette naissance ethnique, et expliquer ces tensions par la volonté d'autonomie du jeune peuple morave par rapport aux Francs?

Peut-être peut-on se contenter d'abord de rappeler brièvement les éléments qui sont communs à ce dernier dossier et au précédent: il sera ainsi plus facile d'exploiter ensuite les spécificités du cas morave. Les traits structurels des échanges sociaux sont les mêmes dans les deux cas. Pas plus que la Pannonie des années 820, la Moravie de la seconde moitié $\mathrm{du} \mathrm{IX}^{\mathrm{e}}$ siècle ne présentait de forte spécificité sociale, religieuse ou matérielle par rapport au monde franc, qui aurait pu favoriser les conflits ; mais comme précédemment, le conflit était géré surtout, du côté carolingien, par un parti local. Et celui-ci, par rapport à l'ensemble des Francs, cultivait une spécificité au moins aussi forte que l'avaient fait les comtes d'Italie naguère: désormais, en effet, c'était aux fils du roi, Carloman puis Arnulf, qu'incombait la gestion des populations slaves voisines ${ }^{23}$. Or ces princes, cherchant à développer des réseaux sociaux pour assurer leur propre accession au trône, cultivaient les intérêts locaux avec un zèle qui tournait parfois à l'opposition envers le pouvoir central, comme le montrent les événements de 861 : «Carloman, le fils aîné du roi [Louis le Germanique], conçut des projets de rénovation: en effet, il chassa les chefs auxquels était confiée la garde de la frontière pannonienne et carinthienne, et confia aux siens la gestion de cette marche. Cela ne fut pas sans heurter l'esprit du roi, qui soupçonnait une rébellion ${ }^{24}$.»

C'étaient de tels groupes locaux, en quête de puissance et de reconnaissance, qui constituaient les principaux acteurs de la politique franque vis-à-vis des Slaves. Or pour eux, le thème d'un peuple slave en quête d'autonomie constituait un argument précieux, qu'il fût réel ou imaginaire : il renforçait en effet le caractère indispensable des défenseurs frontaliers au sein du monde franc, permettant ainsi à Carloman ou à Arnulf de se mettre en valeur et de distribuer des honneurs à leurs hommes. Faut-il en conclure que là encore, ces intermédiaires régionaux ont délibérément inventé ou exagéré, à l'intention des monarques francs, les aspirations ethniques des Moraves?

La violence semblait pourtant bien réelle et alimentée par les deux camps. Et au fil de la seconde moitié $\mathrm{du} \mathrm{IX}^{\mathrm{e}}$ siècle, les sources produisent même une impression d'aggravation dans ces conflits, que les historiens ont mise en relation avec la consolidation de la principauté morave : celle-ci se serait opposée aux Francs avec une résolution croissante ${ }^{25}$. Ainsi, dans les années 850 , les opérations franques en direction de leurs voisins slaves ne consistaient encore qu'en expéditions saisonnières destinées à obtenir du butin ${ }^{26}$; en 870 au contraire, Carloman entreprit une invasion plus durable de la principauté slave, dont il ne fut expulsé que l'année suivante, les combats prenant alors un tour particulièrement brutal ${ }^{27}$; en 884 , ce fut au tour du chef morave Zventibald de s'en prendre à la Pannonie franque, que, «monstrueux et cruel comme un loup, il massacre, il dévaste, [...] il détruit, il incendie ${ }^{28} »$; à l'extrême fin du $\mathrm{IX}^{\mathrm{e}}$ siècle enfin, les ambitions des dirigeants moraves inquiétèrent jusqu'à leurs voisins de Bohême, qui sollicitèrent alors le soutien des Francs ${ }^{29}$. À cette date, les conflits avec les Slaves occupaient l'essentiel du récit dans les Annales de Fulda. Faut-il en déduire que les sentiments d'identité ethnique des Moraves s'étaient exacerbés au cours de ce demisiècle, provoquant des conflits plus fréquents et plus violents?

21 Avant d'en arriver à cette conclusion, il convient de prendre garde, une nouvelle fois, à la nature précise de nos sources. En effet, le texte que l'éditeur a choisi de publier sous le titre unique d'Annales de Fulda est en réalité une œuvre complexe, qui rassemble des 
traditions différentes ${ }^{30}$. Ainsi, jusqu'en 887 , ce récit fut compilé par un proche de l'archevêque de Mayence; mais à partir de 882, un autre auteur, localisé à Ratisbonne, avait repris à son compte le texte qui avait été rédigé jusque-là, le prolongeant dans une autre version jusqu'en 897 ; enfin, passé cette date, ce fut un scribe d'Altaich qui poursuivit le récit jusqu'en 901. Mayence, Ratisbonne, Altaich: on constate que la rédaction de ce texte fut de plus en plus proche de la frontière entre Francs et Moraves. De ce fait, l'importance accrue de la violence dans le récit ressemble fort à un mirage des sources : celles-ci reflétaient avant tout un point de vue de plus en plus local, qui devenait ainsi plus sensible aux violences quotidiennes ou annuelles entre des groupes voisins. Il faut donc se garder de voir dans les événements de cette seconde moitié du siècle le signe d'une hostilité croissante de la part des Moraves ; en revanche, les Francs des périphéries orientales, pour leur part, avaient intérêt à accentuer la rupture, et ce projet influença d'autant plus nos sources que celles-ci étaient rédigées de plus en plus près de la frontière.

Mais si l'on fait abstraction de ce discours-écran, quelles relations les Moraves cherchaient-ils réellement à entretenir avec le pouvoir carolingien? Cette question était insoluble dans le cas de Liudewit, faute de sources contradictoires; en revanche, à propos du prince Zventibald, elle trouve quelques éléments de réponse. Par deux fois, en effet, les Moraves parvinrent à contourner le monopole d'information que détenaient généralement les Francs des frontières, et à faire entendre leur propre voix auprès du souverain ; et ces fragments de vérité éclairent d'un autre jour les difficultés que pouvait poser la gestion des frontières pour les monarques de Francie orientale. Ainsi, en 873, au terme d'un conflit particulièrement acharné entre Carloman et Zventibald, les Annales de Fulda rapportent l'épisode suivant: «[Lors d'un plaid à Francfort] se présenta également devant le roi [Louis le Germanique] un Alaman nommé Berehtram, qui avait été capturé par les Slaves moraves l'année précédente; envoyé par Zventibald, il transmit le message qui lui avait été remis par ce chef, parce qu'il avait promis, sous le sceau du serment, qu'il le ferait ${ }^{31} »$.

Le contenu du message n'était pas indiqué par l'annaliste; en revanche, dès l'année suivante, l'empereur accueillit une ambassade morave complète, qui fut suffisamment convaincante pour aboutir à une paix durable, alors qu'auparavant, la guerre avait fait rage durant plusieurs années ${ }^{32}$. Comment le seul message de Berehtram avait-il permis de rétablir la confiance, et pourquoi avait-on jugé nécessaire de lui faire prêter serment? Précisons que la cause du conflit, plusieurs fois avancée par Carloman, résidait dans les «trahisons » attribuées à Zventibald. Or pour celui-ci, il était difficile de plaider sa cause devant Louis le Pieux : en période de guerre, il n'était en effet guère aisé pour un ambassadeur morave de gagner la cour impériale en évitant les troupes de Carloman. Ce fut manifestement la raison qui avait poussé à recourir aux services d'un prisonnier de guerre, qui présentait l'avantage supplémentaire de ne pas être originaire de cet espace-frontière et d'être donc relativement indépendant face au discours de Carloman. L'épisode permet deux conclusions : d'une part, il illustre le parti pris du discours des Francs frontaliers, puisque les Slaves jugèrent nécessaire de recourir à un tel expédient pour informer Louis le Pieux; d'autre part, il permet de déceler l'orientation réelle de Zventibald, certes hostile au chef local que constituait Carloman, mais soucieux en permanence de préserver l'échange avec le pouvoir impérial carolingien. 

de cet espace périphérique. Dans un document rédigé vers 890, un comte franc, Arbo, envoyait au nouveau roi de Francie orientale, Arnulf, le message suivant dans un latin hésitant : «Au nom de la Trinité sainte et indivise. À Arnulf, roi très serein des Francs par la faveur de la clémence divine, vie et salut. Arbo, votre humble comte et fidèle serviteur. Que votre clémence sache qu'il n'y a pas d'homme libre ni de serviteur (servus) qui lui soit plus fidèle que nous aussi longtemps que je (!) serai valide. Nous faisons parvenir à vos oreilles le fait que nos envoyés sont venus des secteurs orientaux dimanche dernier; ils nous ont rapporté que tous les Moraves avaient envoyé d'un même mouvement du bétail pour payer leur dû (servitium), comme doivent le faire des serviteurs fidèles (proprii); ils respectent l'alliance et le service qu'ils vous doivent, et ils paient sans aucune contrainte leur charge, qui est importante. Seigneur, ils ont accueilli avec joie l'évêque Wiching ${ }^{33}$ et votre autre envoyé, et ils ont nié ce qui leur était imputé. Et tout (...) est sous contrôle et ils les (?) rassemblent chaque jour pour payer ce qui vous est dû. Seigneur très pieux, lorsque vous avez quitté notre secteur (...) j'ai été saisi (...) dans l'espace oriental [les deux dernières lignes du manuscrit, fort incomplètes, résistent à une traduction] ${ }^{34}$ ".

Le texte, on l'aura remarqué, est fragmentaire; ce qui en subsiste suffit toutefois pour éclairer un paradoxe. D'une part, entre Francs et Slaves vers 890, les relations semblaient paisibles, et les prélèvements se déroulaient selon des règles admises de part et d'autre. Mais d'autre part, il existait manifestement un courant d'opinion, qu'Arbo s'efforçait de réfuter, visant à discréditer les Moraves auprès du monarque carolingien. Ce fut manifestement ce parti des faucons qui l'emporta au sein des Francs, puisque les combats reprirent dès l'année 892. Étant donné le texte que l'on vient de présenter, il semble difficile d'admettre l'argumentation des Annales de Fulda, qui attribuaient la guerre, une nouvelle fois, aux trahisons de Zventibald. En effet, quelle que fût la part de bonne foi dans les messages envoyés par celui-ci aux Francs, il semble évident qu'il ne cherchait pas à déclencher un conflit : les heurts militaires dans ces espaces n'illustraient en rien une quelconque évolution sociale des sociétés slaves. orientales carolingiennes n'ont pas attendu la présente étude pour être mis en lumière. Toutefois, on a essayé de suggérer ici que pour les conflits dont ces espaces furent le théâtre, le principal facteur déclenchant ne fut pas un réflexe d'autonomie ethnique au sein des populations slaves : celles-ci, face à l'ensemble culturel franc, contestaient une partie, mais acceptaient le tout. Certes, il se peut qu'elles aient profité de la distance qui les séparait du pouvoir central carolingien, de manière à secouer l'autorité la plus proche - qui est souvent la plus contraignante - tout en reconnaissant un maître lointain qui pouvait difficilement les menacer. Mais de leur côté, les agents francs installés dans ces espaces-frontières ne se privèrent pas d'exploiter également cette même distance face au prince, pour développer leurs intérêts locaux. Dans cette optique, la naissance de peuples slaves au $\mathrm{IX}^{\mathrm{e}}$ fut peut-être une conséquence de ces conflits entre les grands ; il semble improbable qu'elle en ait été la cause.

Si ces relations sociales semblent parfois surprenantes pour l'historien, ce n'est pas seulement parce qu'y apparaissent sans cesse des phénomènes nouveaux qu'il lui faut comprendre, ni parce qu'y règnent des règles qu'il ignore en partie. C'est également parce que le monde carolingien, à l'époque de son expansion maximale, était devenu pour lui-même une société non transparente : faute de pouvoir pleinement maîtriser la 
distance, les décideurs n'étaient pas toujours les mieux informés à propos des données locales. De ce fait, la seule réputation de loyauté comptait parfois bien plus que l'attitude réellement adoptée, et le principal facteur politique, dans les conflits entre Francs, Pannoniens et Moraves, semble avoir résidé dans l'aptitude des uns et des autres à convaincre le souverain. Ce ne fut d'ailleurs pas la dernière fois qu'une guerre éclata en raison d'une incompréhension (savamment entretenue, il est vrai, par les groupes militaires) à propos des objectifs de l'adversaire.

\section{NOTES}

1. E. DÜMMLER, «Über die älteste Geschichte der Slawen in Dalmatien (549-928) », Sitzungsberichte der Österreichischen Akademie der Wissenschaften. Philos.-historische Klasse 20, 1856, p. 353-430 ; P. Šafař́́k, Slovanské starožitnosti, Prague, 1862-1863.

2. R. WensKus, Stammesbildung und - Verfassung. Das Werden der frühmittelalterlichen Gentes, Cologne-Graz, 1961 ; H. WolfRAm, Die Geburt Mitteleuropas: Geschichte Österreichs vor seiner Entstehung, Vienne, 1987.

3. On peut citer, parmi d'autres, P. B ARFORD, The Early Slavs. Culture and Society in Early Medieval Eastern Europe, Ithaca, 2001; F. CURTA, The Making of the Slavs: History and Archeology of the Lower Danube Region col. 500-700, Cambridge - New York - Melbourne, 2001.

4. Annales Regni Francorum, F. KURZE éd., MGH SRG 6, Hanovre, 1895 (désormais abrégé en : ARF), p. 84 .

5. Cf. essentiellement A.B ORST, Der Turmbau von Babel. Geschichte der Meinungen über Ursprung und Vielfalt der Sprachen und Völker, $2^{\mathrm{e}}$ édition Munich, 1995.

6. En dernier lieu, S. BRATHER, Archäologie der westlichen Slawen. Siedlung, Wirtschaft und Gesellschaft im früh- und hochmittelalterlichen Ostmitteleuropa, Berlin - New York, 2001.

7. Un exemple parmi d'autres: R.ERNST, Die Nordwestslawen und das fränkische Reich. Beobachtungen zur Geschichte ihrer Nachbarschaft und zur Elbe als nordöstliche Reichsgrenze bis zur Zeit Karls des Gro

8. S. BRATHER, « Nordwestslawische Siedlungskeramik der Karolingerzeit : fränkische Waren als Vorbild? », Germania n 73 (1995), vol. 2, p. 403-420.

9. Tel était le cas, en particulier, des Avars ou des Bulgares.

10. ARF, a. 822, p. 159.

11. ARF, a. 817, p. 147 , et a. 821 , p. 157.

12. ARF, a. 822, p. 159.

13. ARF, a. 824, p. 165.

14. Annales de Saint-Bertin, F. GRAT, J. VieILlaRd et S. ClémencEt éd., Paris, 1964, p. 30-31.

15. ARF, a. 826, p. 168 et 170 .

16. ARF, a. 819 , p. 151.

17. ARF, a. 820, p. 152.

18. Rappelons par exemple que les Serbes furent nommés pour la première fois en tant que tels en 822 , dans les Annales royales, et que les Croates le furent au plus tôt sous le règne du prince Branimir (879-892). Pour ce dernier point, on pourra se reporter notamment à Ž. Rapanić, «Bilješke uz četiri Branimirova natpisa », Starohrvatska prosvjeta 11 (1981), p. 185-195.

19. H. Wolfram, Die Geburt Mitteleuropas..., op. cit., p. 269. 
20. ARF, a. 818 , p. 149.

21. ARF, a. 819 , p. 150 , et a. 822 , p. 158.

22. Les Annales de Fulda signalent des conflits depuis 847 jusqu'en 900 : Annales Fuldenses, F. KURZE éd., MGH SRG 7, Hanovre, 1891 (désormais abrégé en : AF).

23. K. BRUNNER, Oppositionelle Gruppen im Karolingerreich, Vienne-Cologne-Graz, 1979, p. 141 sq. ; H. WolfRAm, Die Geburt Mitteleuropas..., op. cit., p. 284 sq.

24. AF, a. 861, p. 55.

25. Telle était notamment l'opinion de F. GRAUS, « L'empire de Grand-Moravie, sa situation dans

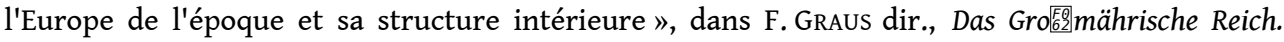
Tagung der wissenschaftlichen Konferenz des archäologischen Instituts der Tschechischen Akademie der Wissenschaften, Prague, 1966, p. 172 sq.

26. Voir par exemple l'expédition de 855 : AF, a. 855, p. 45-46.

27. AF, a. 870 , p. 70 , et a. 871 , p. $73-74$.

28. AF, a. 884 , p. 112 .

29. AF, a. 897 , p. 131 .

30. On résume ici la synthèse historiographique proposée par R. RAU, « Die Annales Fuldenses », dans R. RAU, Quellen zur Geschichte der karolingischen Reichsgeschichte, vol. 3, Darmstadt, 1960, p. 1-177, ici p. 1-5

31. AF, a. 873, p. 78.

32. AF, a. 874 , p. 82 .

33. Celui-ci est connu également par la correspondance du pape Jean VIII, ainsi que par une mention des Annales de Fulda : G. BÜHRER-THIERRY, Évêques et pouvoirs dans le royaume de Germanie. Les Églises de Bavière et de Souabe 879-973, Paris, 1997, p. 183 sq.

34. H. SCHWARZMAIER, «Ein Brief des Markgrafen Aribo an König Arnulf über die Verhältnisse in Mähren », Frühmittelalterliche Studien 6 (1972), p. 55-56.

\section{RÉSUMÉS}

Pour expliquer les conflits qui opposèrent Slaves et Francs au IX siècle, l'historiographie incrimina généralement le processus de consolidation des peuples d'Europe centrale, qui aurait amené ces derniers à rejeter l'influence franque avec une violence croissante. Or plusieurs éléments encouragent à proposer ici une autre hypothèse. En particulier, si l'on examine le rôle des délégués francs près des frontières, on s'aperçoit que, par le monopole d'information dont ils disposaient, ils étaient largement en mesure de définir comme rebelles des populations qui souhaitaient au contraire la préservation de l'échange; or un tel discours correspondait par ailleurs aux intérêts de ces informateurs. C'est pourquoi on propose d'admettre que les conflits naquirent d'une opposition personnelle entre les Slaves et les seuls agents francs qui agissaient en périphérie, et non d'une opposition envers le monde carolingien ou d'une évolution ethnique dans ces secteurs.

To explain the conflicts which opposed Slavs and Franks during the Ixth Century, historiography generally accused the consolidation of Central Europe's ethnic groups, which would have led the latter to reject the Frankish influence with an increasing violence. However, several elements encourage to propose another hypothesis. In particular, if one looks at the role of the Frankish 
agents close to the borders, one realises that, through their monopoly of information, they were largely able to define as rebels some populations who wished on the contrary to keep an exchange; and such a discourse corresponded to these informator's aims. This is why one proposes to admit that the conflicts were born from a personal opposition between the slavs and some Frankish agents acting on the periphery, and not from an opposition towards the Carolingian world or from an ethnic evolution in these sectors.

\section{INDEX}

Mots-clés : identité ethnique, Slaves, Carolingiens, archéologie, Moravie, information

Keywords : ethnic Identity, Slavs, Carolingians, archeology, Moravia, information

\section{AUTEUR}

\section{THOMAS LIENHARD}

LAMOP, Université de Paris I-Sorbonne, 17, rue de la Sorbonne, 75005 Paris 